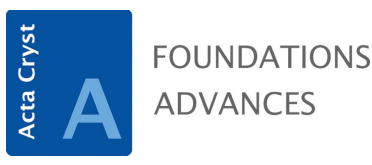

ISSN 2053-2733
Keywords: book review; aperiodic crystals; almost periodicity

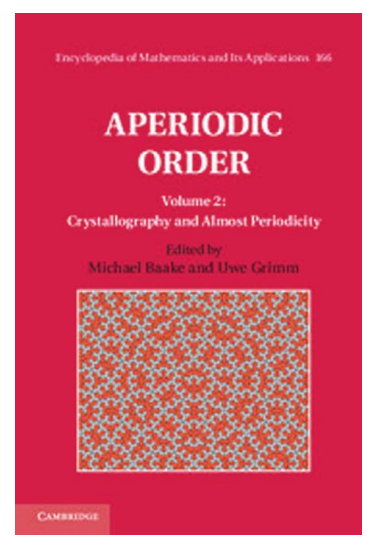

(C) 2019 International Union of Crystallography

\section{Aperiodic Order: Volume 2, Crystallography and Almost Periodicity. Edited by M. Baake and U. Grimm. Cambridge University Press, 2017. Pp. 404. Price GBP 110 (hardback). ISBN 9781139033862.}

\author{
Marc de Boissieu* \\ Université Grenoble Alpes, CNRS, SIMaP, F-38000 Grenoble, France. *Correspondence e-mail: \\ marc.de-boissieu@simap.grenoble-inp.fr
}

This volume is the second in a series presenting the mathematical aspects and theory of aperiodic order as related to quasicrystalline structures.

In crystallography, the notion of aperiodic order arose very soon after the Braggs' discovery of the diffraction of X-rays by periodic crystals, with the discovery of incommensurately modulated structures (incommensurate displacive or compositional modulations, incommensurate magnetic structures). They were interpreted using the beautiful invention of superspace crystallography by P. M. de Wolff, A. Janner \& T. Janssen [see Janssen \& Janner (2014) for a historical perspective]. This was followed by the discovery of incommensurate composite structures and finally quasicrystals.

This area of research has prompted much activity in mathematics from various perspectives, with the aim of establishing a mathematical definition of a 'long-range ordered' structure and what the different kinds of point distributions or tiling are that give rise to diffraction patterns with a pure point spectrum (known as Bragg peaks in crystallography). This is still an open and fascinating field that continues to develop, and is presented in a coherent manner in the book series edited by M. Baake and U. Grimm. The first volume is an introduction to the field of the mathematical aspects of quasicrystals, with all the necessary mathematical tools. This second volume is dedicated to more specialized topics, in particular the notion of 'almost periodicity'.

An introduction by J. C. Lagarias sets the scene for the six chapters that follow. The first presents an extension of tilings obtained by inflations, the second tackles the problems of the mathematical concept of discrete tomography (which differs from the usual tomography techniques), the third deals with the enumeration of lattices in $\mathbb{Z}$-modules, the fourth details the notion of almost periodicity and its Fourier transforms, the fifth introduces the pure point measure of almost periodic functions, and the sixth presents some averaging theorems for almost periodic functions. An epilogue written by P. Kramer reviews the history of the discovery of quasicrystals, both from the experimental and mathematical perspectives.

Although most of the chapters contain an easy-to-read introduction that explains the goal and the problems to be solved, the book is clearly written for mathematicians interested by this growing field.

\section{References}

Janssen, T. \& Janner, A. (2014). Acta Cryst. B70, 617-651. 